\title{
Dimensional resonances in wide parabolic quantum wells
}

\author{
A. Wixforth \\ Sektion Physik, Universität München, D-8000 München 22, Federal Republic of Germany \\ M. Sundaram, K. Ensslin, J. H. English, and A. C. Gossard \\ Materials Department, University of California, Santa Barbara, California 93106
}

(Received 2 January 1991)

\begin{abstract}
The direct observation of dimensional resonances in wide parabolic quantum wells is reported. Using a grating-coupler technique, we are able to excite and detect these resonances without the mediation of a magnetic field. The ability to tune electrically the carrier density in the well and-though not independently - the shape of the confining potential, enables us to compare directly our results with those of a recent theoretical prediction.
\end{abstract}

Remotely doped parabolic quantum wells (PQWs) recently have been used to produce ${ }^{1,2}$ and study ${ }^{3-7}$ wide $(\leq 600 \mathrm{~nm}$ ) electron systems with high electron mobilities. The basic idea behind these structures is to create a conduction-band profile $E_{c}(z)$ in the growth direction of $\mathrm{GaAs}-\mathrm{Al}_{x} \mathrm{Ga}_{1-x} \mathrm{As}$ heterostructures by properly varying the Al mole fraction $x$ such that it mimics the parabolic potential of a uniformly distributed slab of positive charge. This pseudocharge then would have a density $n^{+}$ which is proportional to the curvature of the grown parabolic potential

$$
n^{+}=\frac{\epsilon \epsilon_{0}}{e^{2}} \frac{\partial^{2} E_{c}}{\partial z^{2}}=\frac{8 \epsilon \epsilon_{0} \Delta}{e^{2} W^{2}} .
$$

Here, $\epsilon$ denotes the mean dielectric constant of $\mathrm{Al}_{x}$ $\mathrm{Ga}_{1-x} \mathrm{As}, \Delta$ is the energetic height of the parabola from its bottom to the edges, $e$ the electronic charge, and $W$ the width of the PQW. Remote doping releases electrons into the well, which in turn act to screen the potential of the pseudocharge $n^{+}$, and thus form a wide layer of highly mobile carriers. Self-consistent subband calculations ${ }^{8,9}$ as well as initial experiments ${ }^{3,4}$ have indeed proven that such structures can be realized. Apart from the basic interest in this system, revealing an approach to the theoretical construct of jellium, ${ }^{10,11}$ the study of electrons in parabolic confining potentials has attracted very much attention in the recent past. Here, we mention the realization and investigation of so-called quantum wires and quantum dots, ${ }^{12,13}$ where the confining potentials in most cases can be regarded as parabolic. Theoretically, parabolic confining potentials are also very attractive, since they allow many properties to be calculated in a rigorous and analytical fashion. ${ }^{14,15}$ One of the most interesting recent theoretical results ${ }^{16}$ is that carriers confined in a truly parabolic potential only absorb light at the bare harmonicoscillator frequency $\omega_{0}$, independent of the number of electrons in the well. In other words, the electron-electron interactions do not affect the absorption of radiation of such systems, a fact which can be considered as a generalization of Kohn's theorem. ${ }^{17}$ For a PQW as described above, this harmonic oscillator frequency is given by

$$
\omega_{0}=\left(\frac{8 \Delta}{W^{2} m^{*}}\right)^{1 / 2},
$$

which is by construction equal to the plasma frequency of a three-dimensional (3D) electron gas of density $n^{+}$and effective mass $m^{*}$. Recent experiments indirectly prove this behavior by coupling the cyclotron resonance $\hbar \omega_{c}$ in tilted or parallel magnetic fields to the collective dimensional resonance $\hbar \omega_{0}$ of the electrons in PQWs. ${ }^{18-20}$ The direct observation of this resonance as a function of the number of carriers in the well, however, was lacking.

Here, we like to present results of our recent experiments, in which we use a grating-coupler technique ${ }^{21}$ to excite directly dimensional resonances in PQWs. The samples used in our experiments are grown by molecularbeam epitaxy (MBE) and have widths $W$ between 75 and $568 \mathrm{~nm}$. The growth procedure has been described in detail before. ' By application of a front-gate bias we are able to tune simultaneously the carrier concentration and, employing specially designed samples, also the shape of the external confining potential. ${ }^{6}$ Here, we will focus on two samples, namely $\mathrm{S} 1$, a nominally 75-nm-wide PQW with $\Delta=75 \mathrm{meV}$, and $\mathrm{S} 2$, with $W=200 \mathrm{~nm}$ and $\Delta=150$ $\mathrm{meV}$. Both wells have vertical sidewalls, as depicted in Fig. 1. The curvature of $\mathrm{S} 1$ is chosen such that we expect the bare harmonic oscillator frequency to be $\hbar \omega_{0}=86$ $\mathrm{cm}^{-1}$, whereas $\mathrm{S} 2$ is designed for $\hbar \omega_{0}=47 \mathrm{~cm}^{-1}$. As it turns out, in S1 we can electrically switch between a nearly perfect parabola and a potential that contains strong nonparabolic contributions. This way we can investigate the influence of deviations from parabolicity on the farinfrared (FIR) spectrum of the sample. In contrast, on sample $\mathrm{S} 2$ we observe that for all achievable carrier densities the harmonic-oscillator model remains valid.

To induce longitudinal electric components in the FIR radiation, that are transmitted through the sample parallel to the growth direction, we use a Ag grating coupler of periodicity $d=6 \mu \mathrm{m}$ that is deposited on top of the sample. In addition, a semitransparent 5-nm Ni-Cr front-gate electrode is used to change the carrier density in the well. Au-Ge contacts are alloyed at $T=430^{\circ} \mathrm{C}$ to provide Ohmic contacts to the electron system. Our experiments are carried out in transmission at liquid-helium temperatures using a rapid-scan Fourier-transform spectrometer. Experimentally, we determine the relative change in transmission $-\Delta T / T=\left[T(0)-T\left(N_{s}\right)\right] / T(0)$, which is proportional to the real part of the conductivity $\tilde{\sigma}_{z z}(\omega)$ 


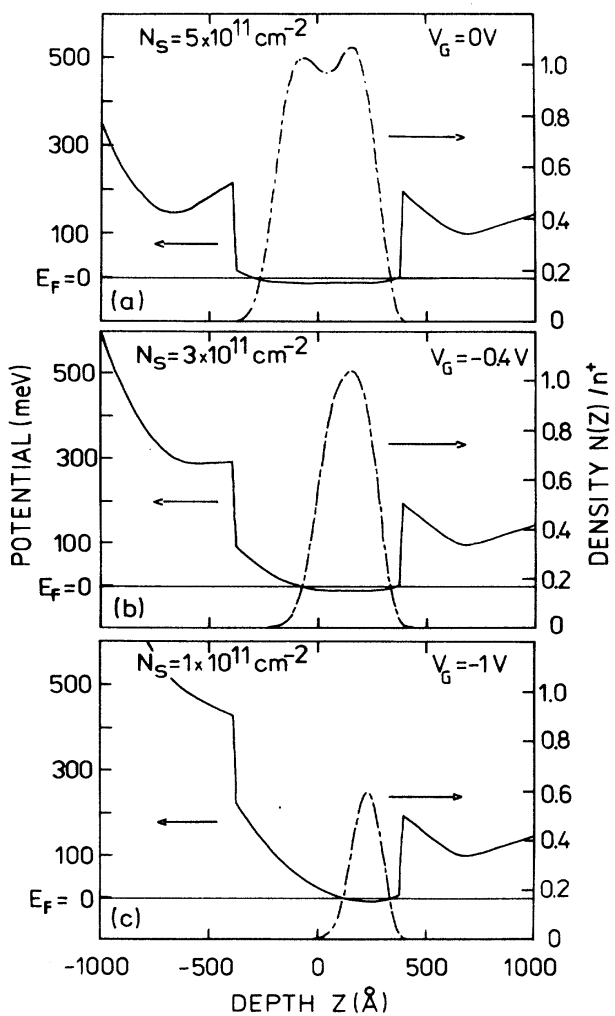

FIG. 1. Results of a self-consistent subband calculation for the sample $\mathrm{S} 1(W=75 \mathrm{~nm}, \Delta=75 \mathrm{meV})$. Both the self-consistent potential as well as the resulting charge distribution $N(z)=\sum_{i} N_{s}^{i}\left|\xi_{i}\right|^{2}$ are depicted as a function of depth $z$. The center of the MBE grown parabolic well is located at $z=0$. Note the considerable shift of the electron distribution with increasing negative gate bias. In (a) we show the situation for the complete filled well, (b) depicts intermediate filling, whereas in (c) the well is nearly depleted.

normal to the plane of the well. ${ }^{22} T(0)$ is the transmission of the sample with the well being completely depleted, $T\left(N_{s}\right)$ the one at finite carrier densities.

Application of a negative gate bias $V_{G}$ tends to deplete the well as is depicted in Fig. 1. Here, we show the results of our self-consistent subband calculation, ${ }^{6}$ i.e., the potential and the density distribution $N(z)=\sum_{i} N_{s}^{i}\left|\xi_{i}\right|^{2}$ for $\mathrm{S} 1$ and three different carrier densities $N_{s}$. $N_{s}^{i}$ here denote the carrier densities in the different subbands and $\xi^{i}$ the corresponding wave functions, respectively. With increasing $N_{s}$, the filled part of the well becomes wider and flatter, approaching the ideal case of a wide square well. With increasingly negative gate bias the minimum of the potential shifts deeper into the material. Although the curvature of the parabola is of course unaffected by the application of the bias, the shift of the electron wave functions closer to the edge causes an increase in the deviation from parabolicity [cf. Fig. 1(c)].

In Fig. 2 we show the experimental traces for the sample $\mathrm{S} 1$. Here, we plot the relative change in transmission versus FIR frequency for different gate voltages $V_{G}$. Capacitance-voltage measurements ${ }^{6}$ on the same sample reveal a threshold voltage of $V_{\text {th }}=-1.2 \mathrm{~V}$ for complete

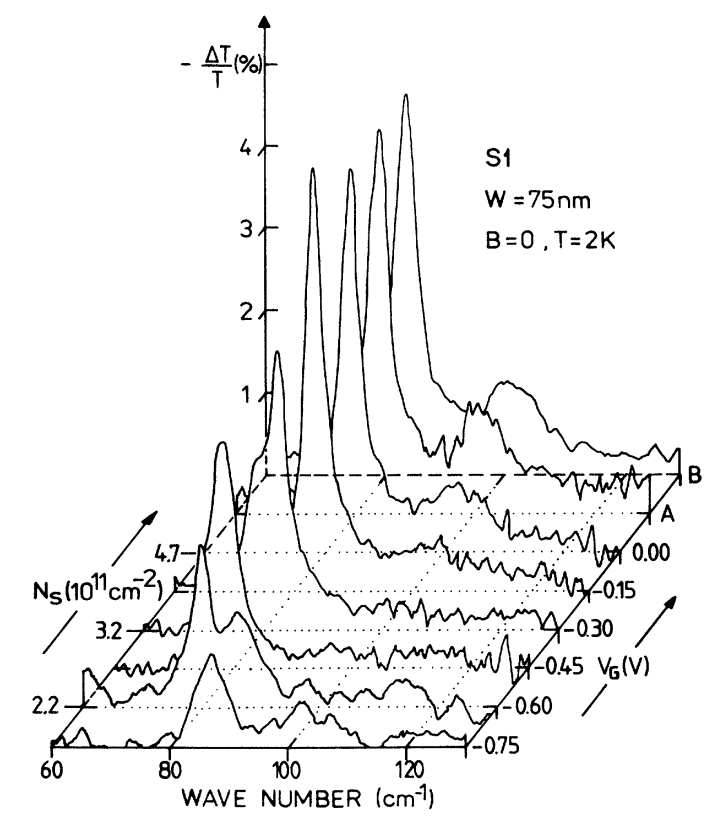

FIG. 2. Experimentally obtained spectra for sample S1. The relative change in transmission $-\Delta T / T$ is shown for different gate voltages $V_{G}$. Traces $A$ and $B$ have been taken after a short illumination of the sample $\left[V_{G}(A)=0.0 \mathrm{~V}, V_{G}(B)=+0.6 \mathrm{~V}\right]$, which still increases the carrier density in the well with $N_{s}(A)<N_{s}(B)$. In both the limits of high and low electron concentration deviations from the harmonic-oscillator picture are observed, manifesting themselves in the occurrence of more than one absorption line.

depletion. Trace $A$ has been recorded after illuminating the sample for some seconds with an IR light-emitting diode, thus increasing the carrier concentration via the persistent photoeffect. Trace $B$ is taken at a gate bias of $V_{G}=+0.6 \mathrm{~V}$ after illumination which further increases $N_{s}$. The exact values for the densities at $A$ and $B$, however, have not been determined in the experiment but from an analysis of the oscillator strength of the resonance $N_{s}(B)$ is estimated to be of the order of $6 \times 10^{11} \mathrm{~cm}^{-2}$. As can be seen from Fig. 2, in this special sample the electron system not only absorbs at the frequency of the bare harmonic potential, but sidelines appear in the spectrum. The influence of nonparabolic contributions has recently been discussed in considerable detail by Brey et al. ${ }^{23}$ They calculate the IR absorption of what they call "imperfect" parabolic wells using the local density approximation for a PQW similar to the ones we use in our experiments. For comparison, in Fig. 3 we replot the result of Brey and co-workers for a completely filled well, where finite-size effects become important, together with trace $B$ from Fig. 2. Clearly all essential features of the experiment are reproduced in the calculation, although the linewidths and the exact position of the satellite structure differ. This is believed to be due to the fact that the sample assumed in the calculation is not identical with ours and that a phenomenological scattering time $\tau$ has been used.

Sidelines do not appear for the wider and deeper well $\mathrm{S} 2$ where we indeed deal with a nearly perfect parabolic 


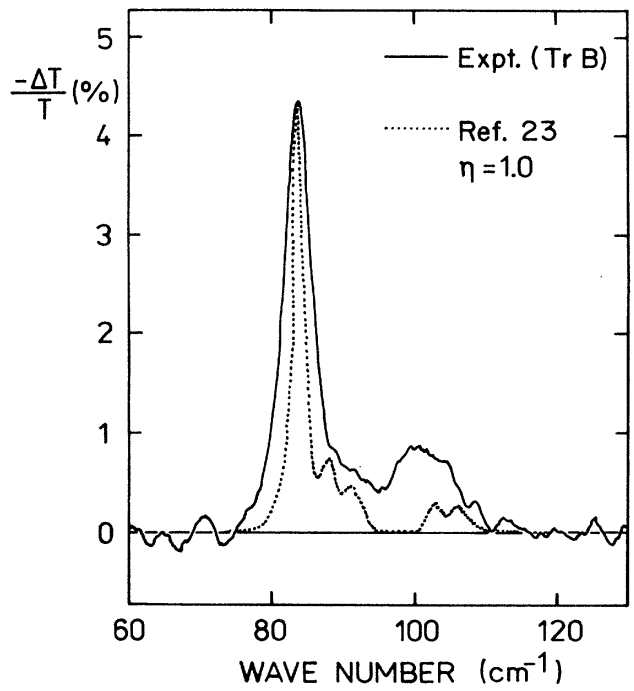

FIG. 3. Comparison of the experimentally obtained resonances of trace $B$ in Fig. 2 with those calculated by Brey and co-workers for a completely filled PBW. The sidelines are due to internal oscillations of the electron system caused by the finite width of the well. All essential features of the experiment are reproduced in the calculation of the far-infrared absorption. The deviations are believed to occur due to differences in the sample parameters in both cases and the uncertainty of the exact filling factor $\eta$ in the experiment.

potential, since here finite-width effects play only a minor role. Experiments using tilted and parallel magnetic fields ${ }^{20}$ on the same samples confirm our observations at $B=0$. The detailed interpretation of the magnetic-field spectra, however, is quite complex and will be discussed elsewhere.

In Fig. 4 we depict the observed resonance positions for both samples as a function of the applied gate bias. The dashed lines in the figures represent the expected resonance positions as derived from the growth parameters alone. For sample S1 [Fig. 4(a)] we are able to more or less intentionally tune the degree of nonparabolicity. This special well is quite narrow and shallow, so that the application of an even moderate gate bias has a large effect on the actual potential shape and the position of the wave functions, as can be seen in Fig. 1. Moreover, the doping is such that we are able to completely fill the well, or even slightly overfill it.

According to Brey and co-workers for a filling factor $\eta=N_{s}\left(n^{+} W\right) \approx 1$ the perturbation has two main effects on the IR absorption of a PQW. First, the additional confinement leads to a slight shift of the main absorption line to a higher frequency and second, small satellites begin to appear, reflecting the coupling of light to the internal oscillations of the electron system. As can be seen in Fig. 2, this crossover is clearly discernable in our data. At high $\eta$ (see also Fig. 3) two lines are clearly observed. The one of smaller oscillator strength and of higher frequency disappears with decreasing $N_{s}$. At intermediate

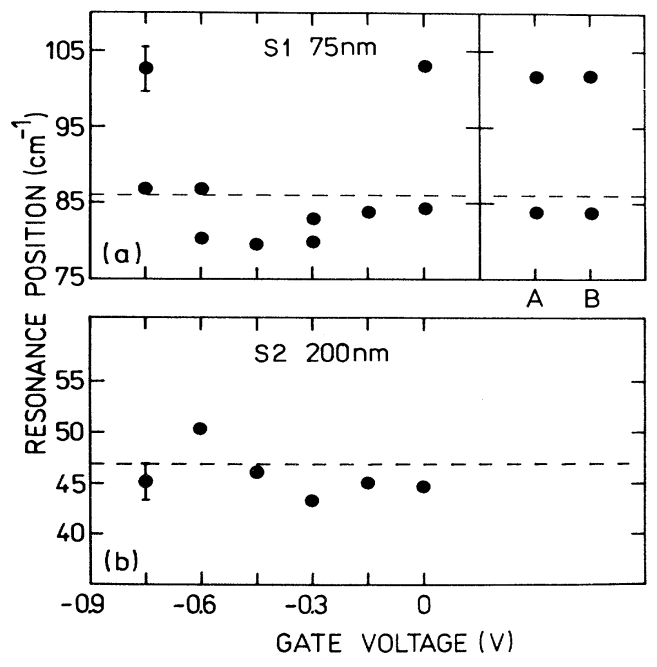

FIG. 4. Observed resonance positions as a function of the applied gate voltage. In (a) the result for sample $S 1(W=75 \mathrm{~nm}$, $\Delta=75 \mathrm{meV})$ and in (b) the result for $\mathrm{S} 2(W=200 \mathrm{~nm}, \Delta=150$ $\mathrm{meV})$ are shown. The narrower sample can be electrically tuned into various deviations from parabolicity as described in the text, leading to the occurrence of satellite structures. Sample S2 is closer to the ideal case of a perfect parabola. The dashed lines represent the expected resonance positions from growth in the harmonic-oscillator picture.

densities only one resonance is observed until at low $N_{s}$ the system begins to feel the strong deformation of the potential associated with the vertical confining sidewall [cf. Fig. 1(c)]. Here, the model of the harmonic oscillator is certainly not valid anymore and as a consequence, additional resonances appear. Experiments in a magnetic-field confirm this observation at $B=0$. They also indicate that for a correct theoretical description, the matrix elements for the transitions have to be properly adjusted to the experimental situation.

For the ideal sample S2 [Fig. 4(b)] the resonances scatter around the expected value, with a distribution of approximately $\delta \omega / \omega=5 \%$. The same qualitative behavior again is observed in magnetic fields under various tilt angles, where we deduce nearly the same resonance positions for corresponding $N_{s}$. We believe this reproducible "scatter" represents a very sensitive test of the local curvature of the PQW. Since the positions of the resonances crucially depend on the actual shape of the bare potential, small deviations from a constant curvature have a large impact on the resonance positions. In their theoretical analysis, Brey and co-workers also studied the influence of small additional quartic terms $\Delta_{q}$ in the potential as a measure for deviations from parabolicity. They found that such a correction is quite effective in shifting the absorption line. For example, a positive $\Delta_{q}$ causes a convex parabolic correction to the nearly uniform charge distribution produced by the parabolic potential alone. This leads to an additional confinement of the carriers and thus 
to an increase of the resonance position. A negative $\Delta_{q}$, however, induces an additional concave parabolic component, leading to a spreading of the charge as compared to the ideal parabola and thus to a reduction of the resonance position. A rough estimate yields that a shift of the absorption line by $5 \%$ could be caused by quartic contributions to the potential of the order of $\Delta_{q} / \Delta \approx 0.1$.

In conclusion, dimensional resonances in wide PQWs have been directly observed by use of a grating-coupler technique. Our experiments demonstrate the applicability of the generalized Kohn theorem as derived in Ref. 16, and show that ideally behaving parabolic confining potentials can be realized only under certain conditions. In a specially designed sample, we are able to tune the shape of the external potential such that we can study the influence of various deviations from parabolicity on the absorption spectrum of the sample. Our experimental observations can be well explained in terms of self-consistent calculations of the potential ${ }^{6}$ together with the results of a numerical study ${ }^{23}$ on imperfections in wide parabolic wells.

We would like to thank R. M. Westervelt, E. G. Gwinn, E. Batke, and J. P. Kotthaus for fruitful discussions. This work was sponsored in part by U.S. Air Force Office of Scientific Research under Contract No. AFOSR-88-099, and in part by the Volkswagen Stiftung. We gratefully acknowledge this financial support.
${ }^{1}$ M. Sundaram, A. C. Gossard, J. H. English, and R. M. Westervelt, Superlatt. Microstruct. 4, 683 (1988).

${ }^{2}$ M. Shayegan, T. Sajoto, M. Santos, and C. Silvestre, Appl. Phys. Lett. 53, 791 (1988).

${ }^{3}$ T. Sajoto, J. Jo, M. Santos, and M. Shayegan, Appl. Phys. Lett. 55, 1430 (1989).

${ }^{4}$ E. G. Gwinn, R. M. Westervelt, P. F. Hopkins, A. J. Rimberg, M. Sundaram, and A. C. Gossard, Phys. Rev. B 39, 6260 (1989).

${ }^{5}$ M. Shayegan, T. Sajoto, J. Jo, M. Santos, and H. D. Drew, Phys. Rev. B 40, 3476 (1989).

${ }^{6}$ A. Wixforth, M. Sundaram, K. Ensslin, J. H. English, and A. C. Gossard, Appl. Phys. Lett. 56, 454 (1990).

${ }^{7}$ A. Wixforth, M. Sundaram, D. Donnelly, J. H. English, and A. C. Gossard, Surf. Sci. 228, 489 (1990).

${ }^{8}$ T. Sajoto, J. Jo, H. P. Wei, M. Santos, and M. Shayegan, J. Vac. Sci. Technol. B 7, 311 (1989).

${ }^{9}$ A. J. Rimberg and R. M. Westervelt, Phys. Rev. B 40, 3970 (1989).

${ }^{10}$ B. I. Halperin, Jpn. J. Appl. Phys. 26, 1913 (1987).

${ }^{11}$ V. Celli and N. D. Mermin, Phys. Rev. 140, A839 (1965).
${ }^{12}$ W. Hansen, M. Horst, J. P. Kotthaus, U. Merkt, Ch. Sikorski, and K. Ploog, Phys. Rev. Lett. 58, 2586 (1987).

${ }^{13}$ Ch. Sikorski and U. Merkt, Phys. Rev. Lett. 62, 2164 (1989).

${ }^{14}$ V. Fock, Z. Phys. 47, 446 (1928).

${ }^{15}$ See, e.g., J. C. Maan, in Two Dimensional Systems: Heterostructures and Superlattices, edited by G. Bauer, F. Kuchar, and H. Heinrich (Springer, Berlin, 1984), p. 183.

${ }^{16}$ L. Brey, N. F. Johnson, and B. I. Halperin, Phys. Rev. B 40, 10647 (1989).

${ }^{17}$ W. Kohn, Phys. Rev. 123, 1242 (1961).

${ }^{18}$ K. Karrai, H. D. Drew, H. W. Lee, and M. Shayegan, Phys. Rev. B 39, 1426 (1989).

${ }^{19}$ K. Karrai, X. Ying, H. D. Drew, and M. Shayegan, Phys. Rev. B 40, 12020 (1989).

${ }^{20}$ A. Wixforth, M. Sundaram, J. H. English, and A. C. Gossard (unpublished).

${ }^{21}$ E. Batke, G. Weimann, and W. Schlapp, Phys. Rev. B 39, 11171 (1989).

${ }^{22}$ E. Batke and D. Heitmann, Infrared Phys. 24, 189 (1984).

${ }^{23}$ L. Brey, J. Dempsey, N. F. Johnson, and B. Halperin, Phys. Rev. B 42, 1240 (1990). 